EPJ Web of Conferences 70, 00047 (2014)

DOI: $10.1051 /$ epjconf/ 20147000047

(C) Owned by the authors, published by EDP Sciences, 2014

\title{
High-energy cosmic rays and tests of basic principles of Physics
}

\section{Looking at the Planck scale and beyond}

\author{
L. Gonzalez-Mestres ${ }^{a}$ \\ ${ }^{1}$ LAPP, Université de Savoie, CNRS/IN2P3 \\ B.P. 110, 74941 Annecy-le-Vieux Cedex, France
}

\begin{abstract}
With the present understanding of data, the observed flux suppression for ultra-high energy cosmic rays (UHECR) at energies above $4.10^{19} \mathrm{eV}$ can be a signature of the Greisen-Zatsepin-Kuzmin (GZK) cutoff or be related to a similar mechanism. But it may also correspond, for instance, to the maximum energies available at the relevant sources. In both cases, violations of special relativity modifying cosmic-ray propagation or acceleration at very high energy can potentially play a role. Other violations of fundamental principles of standard particle physics (quantum mechanics, energy and momentum conservation, vacuum homogeneity and "static" properties, effective space dimensions, quark confinement...) can also be relevant at these energies. In particular, UHECR data would in principle allow to set bounds on Lorentz symmetry violation (LSV) in patterns incorporating a privileged local reference frame (the "vacuum rest frame", VRF). But the precise analysis is far from trivial, and other effects can also be present. The effective parameters can be related to Planckscale physics, or even to physics beyond Planck scale, as well as to the dynamics and effective symmetries of LSV for nucleons, quarks, leptons and the photon. LSV can also be at the origin of GZK-like effects. In the presence of a VRF, and contrary to a "grand unification" view, LSV and other violations of standard principles can modify the internal structure of particles at very high energy and conventional symmetries may cease to be valid at energies close to the Planck scale. We present an updated discussion of these topics, including experimental prospects, new potentialities for high-energy cosmic ray phenomenology and the possible link with unconventional pre-Big Bang scenarios, superbradyon (superluminal preon) patterns... The subject of a possible superluminal propagation of neutrinos at accelerator energies is also dealt with.
\end{abstract}

\section{Introduction}

It is not clear by now [1, 2] whether UHECR flux suppression above $\simeq 4.10^{19} \mathrm{eV}[3,4]$ is a signature of the Greisen-Zatsepin-Kuzmin (GZK) cutoff [5, 6] or corresponds, for instance, to the maximum energies available at astrophysical sources or even to an alternative flux

a e-mail: $\operatorname{lgm} \backslash$ _sci@yahoo.fr

This is an Open Access article distributed under the terms of the Creative Commons Attribution License 2.0, which permits unrestricted use, distribution, and reproduction in any medium, provided the original work is properly cited. 


\section{EPJ Web of Conferences}

suppressing mechanism of a different dynamical origin. All these phenomena can be sensitive, for instance, to violations of standard special relativity modifying cosmic-ray production $[7,8]$, propagation $[9,10]$ or acceleration $[11,12]$ at very high energy. Data from UHECR experiments are expected to allow to obtain bounds on Lorentz symmetry violation (LSV) parameters in models incorporating a privileged local reference frame that we call the "vacuum rest frame" (VRF [13, 14]). But the precise phenomenological analysis of experimental data is complicated by the possible presence of other new mechanisms of similar nature, including violations of other fundamental principles of standard physics. Existing low-energy bounds on LSV [15] must also be taken into account in energy dependent LSV patterns.

The effective parameters of such new phenomena can be directly related to Planck-scale physics or to physics beyond Planck scale [16], as well as to the precise LSV dynamics and to the effective symmetries relating the LSV mechanisms for the different objects considered (nucleons, quarks, leptons, photon) [17, 18]. Assuming the existence of a VRF, ultra-high energy (UHE) particles are not the equivalent of particles at rest. LSV and other new physics generated at the Planck scale or beyond can modify the internal structure and interaction properties of particles at very high energy $[12,18]$. Then, particle conventional internal structures and symmetries may cease to be valid at energies close enough to the Planck scale. Other possible violations of commonly admitted fundamental principles and standard basic hypotheses including quantum mechanics, energy and momentum conservation, quark confinement, vacuum homogeneity and "static" properties, vacuum dynamics at very small distance scales, effective space dimensions, quark confinement... can also potentially be tested in a long-term program of high-energy cosmic-ray experiments $[12,19]$. Even below ultrahigh energy (UHE), the appearance of exotic signatures cannot be excluded [20, 21]. From a cosmological point of view, possible UHE violations of standard fundamental principles can possibly be related to pre-Big Bang scenarios $[22,23]$ where the new physics would be expected to play a leading role.

In this contribution, we present an updated discussion of UHECR data and phenomenology, paying special attention to unconventional theoretical motivations and to alternatives to standard interpretations of data. As an example, a possible explanation of the observed UHECR spectrum and composition was provided in [22], where the GZK cutoff would be replaced by spontaneous emission of photons or $\mathrm{e}^{+} \mathrm{e}^{-}$pairs in a simple LSV pattern. Other scenarios for UHECR can also be considered, as discussed in [12, 18]. As the OPERA claim [24] on a possible superluminal propagation of the muon neutrino had not yet been withdrawn [43] when this conference was announced, we also briefly discuss the consistency problems $[25,26]$ of $\mathrm{a} \simeq 2.5 \times 10^{-5}$ critical speed anomaly for light neutrinos including the muon neutrino.

\section{UHECR and relativity}

The relativity principle formulated by Henri Poincaré [27] to account for the result of the Michelson-Morley experiment replaced the Galilean principle by a new one adapted to the experimental situation. The speed of light $c$ became then a universal critical speed and the covariance of the Maxwell equations was guaranteed. Electromagnetism is by now a fundamental interaction of standard elementary particles, and Poincaré's relativity has not yet been disavowed by data. But as early as 1921, Albert Einstein [28] pointed out that standard relativity may be broken beyond some very small distance scale where matter would exhibit a different behavior. More than ninety years later, this deep remark remains still valid. 
Crucial for the generation of the main LSV signatures discussed here is the existence of a VRF $[12,13]$. The old ether is then replaced by a suitably LSV-modified version of the vacuum of quantum field theory (QFT) where fields can condense through mechanisms first developed in condensed matter physics (superfluidity, superconductivity...), and where new physics beyond QFT can also manifest itself.

For small wave vectors, the phonons and other objects of solid state physics naturally obey Lorentz-like symmetries with critical speeds of the order of that of sound [13, 14]. The corresponding dispersion relations are deformed at higher frequencies, as the wavelength becomes closer to the space scale characteristic of the inner structure of the matter where phonons and other excitations are generated. The privileged rest frame and the primordial distance scale (f.i. a lattice spacing) manifest themselves through this deformation of the dispersion relation. In our Universe, the Planck scale or some other fundamental scale can play a role similar to that of the lattice spacing in a solid. Thus, the hypothesis of the existence of a local VRF at any space-time position of our present Universe appears perfectly legitimate from a conceptual point of view. The Earth is assumed to move slowly with respect to this VRF. Such a pattern is fundamentally different from a "perfect" space-time symmetry that would be obtained by just deforming the Poincaré algebra of special relativity without really breaking the symmetry and without generating a VRF. In the absence of a VRF, the new symmetry can still prevent the generation of the strongest LSV signatures.

Possible signatures of LSV with a VRF do not only concern the behavior of conventional particles. For instance, if objects with a critical speed $c_{s} \gg c$ (superbradyons [13, 23]) can exist in our Universe as free particles with speeds larger than $c$, they are expected to spontaneously emit "Cherenkow" radiation in the form of standard particles. If the superbradyon energy is high enough, some of these emissions can even be UHECR [7, 8]. The GZK cutoff could then be naturally avoided. This phenomenon would be similar to the decay of more conventional superheavy objects, as considered later by other authors [29]. Free superbradyons can also be a residual signature from a pre-Big Bang scenario where the Planck scale itself may no longer make sense.

Similarly, UHECR can by themselves be at the origin of observable effects generated dynamically at the Planck scale or beyond it [9, 12], and potentially allowing (together with WMAP [30] and Planck [31] data, see [22, 23]) for a study of the new physics and cosmology associated to such scales. A key point for the exploration of possible new physics in UHECR studies is the smallness of the mass term of a cosmic ray at ultra-high energy, making the UHECR interaction and propagation properties sensitive to very small perturbations impossible to detect at lower energy scales.

If a VRF exists, a small LSV can suppress the conventional GZK cutoff through a modification of kinematical balances in the collisions between UHECR and cosmic microwave background photons $[9,10]$. Similarly, unstable particles can become stable at ultra-high energy or new spontaneous decays be generated [32, 33]. UHECR cross-sections with particles at rest can also be modified or even suppressed [34], potentially invalidating the Froissart bound [35] at high enough energy or distorting the measurement of the UHECR flux. The validity of the conventional parton model at such energies is to be reconsidered [36] if the constituents must travel at a common speed (that of the particle) and remain thus confined.

\subsection{UHECR and the fundamental scale}

A simple indicative illustration concerning the potential sensitivity of UHECR to a fundamental scale is obtained taking for UHE particles, with LSV and a VRF, the quadratically 


\section{EPJ Web of Conferences}

deformed dispersion relation $[9,10]$ :

$$
E \simeq p c+m^{2} c^{3}(2 p)^{-1}-p c \alpha\left(p c E_{a}^{-1}\right)^{2} / 2
$$

where $E$ is the energy, $p$ the momentum, $c$ the speed of light, $m$ the mass, $\alpha$ a constant standing for the deformation strength and $E_{a}$ the fundamental energy scale at which the deformation is assumed to be generated.

It can then be readily checked from (1) that the (negative and increasing with energy) deformation term:

$$
\Delta E \simeq-p c \alpha\left(\begin{array}{ccc}
p & \left.c E_{a}^{-1}\right)^{2} / 2
\end{array}\right.
$$

becomes larger than the (positive and decreasing with energy) mass term $m^{2} c^{3}(2 p)^{-1}$ above a transition energy $E_{\text {trans }}$ given by [10, 18]:

$$
E_{\text {trans }} \simeq \alpha^{-1 / 4}\left(E_{a} m\right)^{1 / 2} c
$$

For a proton, with $\alpha=1, E_{\text {trans }} \simeq 10^{20} \mathrm{eV}$ if $E_{a} \simeq 10^{3} E_{\text {Planck }}$ where $E_{\text {Planck }}$ is the Planck energy. A UHECR proton component would then be potentially sensitive to new physics generated three orders of magnitude beyond Planck scale.

For an electron, using (1) and (3), the same effect with $\alpha=1$ would occur at an energy $E_{\text {trans }} \simeq 10^{19} \mathrm{eV}$ if $E_{a} \simeq 2 \times 10^{4} E_{\text {Planck. }}$. A $10^{19} \mathrm{eV}$ muon would similarly be potentially sensitive to physics generated at $E_{a} \simeq 10^{2} E_{\text {Planck }}$.

Above $E \sim E_{\text {trans }}$, we expect the effective internal structure and interaction properties of particles to be modified with respect to the standard relativistic extrapolation from the particle at rest $[12,18]$. New physics generated beyond Planck scale can thus become a basic unconventional ingredient of the actual structure and behaviour of standard elementary particles at energies well below Planck scale.

For nuclei, the situation can be substantially different $[12,18]$. The nucleus can be dealt with, to a first approximation, as an additive set of $N$ nucleons with equal mass carrying a $1 / N$ fraction of momentum. The value of $E_{\text {trans }}$ becomes then $N$ times larger than for protons, and the effective value of $\alpha$ is $N^{2}$ times smaller. A more detailed model for nuclei must take into account the precise values of masses and binding energies, and consider further UHE corrections. The study of the composition of the UHECR spectrum is compulsory to test basic principles through these data.

The deformed dispersion relation for the proton can in turn be modified by its composite structure in terms of quarks and gluons $[12,18]$, leading also in such case to a smaller value of $\alpha$. The standard parton model should then be replaced by a new internal (nuclear-like ?) kinematical structure compatible with the deformation from LSV. Thus, the question of the value of $\alpha$ for quarks, gluons and nucleons, as compared to leptons and to the photon, will also lead to crucial phenomenology.

The quadratically deformed relativistic kinematics (QDRK) used here, in an approach incorporating a VRF and with no exact invariance related to this kinematics, is a phenomenological example of a real LSV where Lorentz symmetry is not replaced by a new space-time symmetry through the deformation. A linearly deformed relativistic kinematics (LDRK), with $\Delta E$ varying quadratically with $p$, would produce too strong effects at low energy for phenomenologically relevant values of UHE parameters [37]. Mixed scenarios with energy thresholds can also be considered [38]. 


\subsection{UHECR acceleration and LSV}

A possible explanation of the observed fall of the UHECR spectrum can be a limitation of the acceleration dynamics at the existing astrophysical sources. In such case, and assuming a very good knowledge of the source properties, bounds on LSV can possibly be obtained [12] taking into account the implications of the suppression of synchrotron radiation predicted by LSV patterns [11]. Such a suppression would potentially allow protons and nuclei to be accelerated to higher energies.

As pointed out in [11], in the case of standard relativistic kinematics a UHE proton with energy:

$$
E \simeq p c+m_{p}^{2} c^{3}(2 p)^{-1}
$$

can emit in the longitudinal direction a photon with energy and momentum $\left(\epsilon, \epsilon c^{-1}\right)$ provided it absorbs an energy $\delta E \simeq m^{2} c^{2} p^{-2} \epsilon / 2$ from the accelerating source. $\delta E$ falls quadratically with the proton energy. LSV drastically changes this situation.

With the QDRK pattern defined by (1) and above the transition scale $E_{\text {trans }}$, we get instead $\delta E \simeq 3 \alpha \epsilon\left(p c E_{a}^{-1}\right)^{2} / 2$. Then, $\delta E$ rises quadratically with the proton energy. At high enough energy, the proton can no longer emit synchrotron radiation except for negligeable values of $\epsilon$. Protons can thus be accelerated to higher energies in the presence of Lorentz symmetry violation and deformed relativistic kinematics (DRK) suppressing synchrotron radiation at UHE.

A new branch of astrophysical tests of LSV can therefore be opened through precise enough studies of acceleration at well-identified sources. Further data and analyses are required to check the usefulness and feasibility of such an approach.

An attempt to set bounds on LSV using synchrotron radiation from the Crab nebula [39] and a LDRK pattern led to $E_{a}>10^{26} \mathrm{GeV}$ with the formula :

$$
E \simeq p c+m^{2} c^{3}(2 p)^{-1}-p^{2} c^{2} E_{a}^{-1}
$$

A similar bound on LSV had been previously obtained in [37] assuming that (5) applies to pions and to the photon, and that the lifetime of a $10^{17} \mathrm{eV}$ neutral pion agrees with special relativity. This is, however, a weak bound for LDRK if one takes into account UHECR properties. Using such a bound as the actual value of $E_{a}$, and the LDRK relation:

$$
E_{\text {trans }} \simeq\left(E_{a} m^{2} c^{4} / 2\right)^{1 / 3}
$$

one would get $E_{\text {trans }} \simeq 3.10^{17} \mathrm{eV}$ for a proton, $\simeq 10^{17} \mathrm{eV}$ for a muon or a pion, and $\simeq 2.10^{15}$ $\mathrm{eV}$ for an electron. These values of $E_{\text {trans }}$ seem too low for realistic phenomenology. Assuming the standard GZK mechanism to be at work with LDRK would require $E_{a} \gtrsim 10^{33} \mathrm{GeV}$.

Analyses of AUGER data have not yet revealed the existence of flux anisotropies allowing to identify specific UHECR sources $[1,2]$.

\section{UHECR, Cosmology and fundamental principles of Physics}

In standard formulations of grand-unified theories, particle symmetries are assumed to become more exact as the energy scale involved increases and the masses of the particles become comparatively smaller. This is not necessarily the case in the LSV patterns considered here, where such an assumption would be valid only below some critical scale of the order of $E_{\text {trans }}$. Above $E_{\text {trans }}$, extrapolations from accelerator results would possibly no longer hold and a new dynamics may become dominant. 


\section{EPJ Web of Conferences}

Remarkably enough [9, 37], above a scale like $E \approx E_{\text {trans }}$, well below Planck scale, new physics generated at the Planck scale or beyond it can manifest itself including possible signatures of an ultimate (superbradyonic ?) composite structure [12, 38] or new space-time properties $[22,23]$. More generally, new properties of conventional particles as well as hidden differences between them not described by standard theories may become apparent at UHE and invalidate lower-energy symmetries and dynamics. This would have strong cosmological implications and clearly lead to a pre-Big Bang approach to the origin of our Universe based in such a new physics.

In Section 2, possible violations of special relativity have been considered. But if a VRF exists, the fact that UHE particles are not the Lorentz equivalent of rest or low-energy particles may have other important consequences. Not only the internal structure of particles can considerably change, but the effective structure and behaviour of vacuum as seen by such particles will depend on the particle structure.

Fluctuations of the vacuum structure at very short distance scales can influence UHECR propagation and interactions. UHECR may be sensitive to vacuum properties beyond standard quantum field theory (SQFT) as considered in $[22,23]$ and previously in the Post Scriptum of [20]. Fluctuations at cosmic scale of the short-distance vacuum structure and behavior can also exist and play a role in UHECR propagation.

Thus, UHECR can be a unique probe to explore the structure of our physical vacuum and to test the validity of all fundamental principles of standard physics including quantum mechanics or energy and momentum conservation $[12,16]$.

Deformed Heisenberg algebras and scenarios with non-commutative space-time have been considered by several authors in the last two decades [40, 41], including string theories [42]. Following the approach suggested in [41], we consider commutation relations between momentum components where the commutators vanish in the zero-momentum limit and become significant at UHE. An example would be:

$$
\begin{aligned}
& \Delta p_{x} \Delta p_{y} \gtrsim \Phi\left(p^{2}\right) \\
& \Delta p_{y} \Delta p_{z} \gtrsim \Phi\left(p^{2}\right) \\
& \Delta p_{z} \Delta p_{x} \gtrsim \Phi\left(p^{2}\right)
\end{aligned}
$$

where $x, y$ and $z$ stand for three orthogonal space directions, and $\Phi(0)=0$. Such commutation relations would naturally suggest at high energy an intrinsic uncertainty $\Delta_{q} E \gtrsim\left[3 \Phi\left(p^{2}\right)\right]^{1 / 2} c$ together with an uncertainty in the direction of the UHECR. These properties would be compatible with: i) a new signature in the UHECR flux faking the GZK cutoff even in the presence of LSV ; ii) a possible failure of the UHECR accelerating sources in the same energy region; iii) the apparent lack of anisotropy of the UHECR flux, even in the presence of identifiable point sources.

Obviously, the possibility that new physics generated at the Planck scale or at a deeper fundamental scale becomes apparent above $\approx E_{\text {trans }}$ provides a major motivation for a longterm experimental effort, ground based and through satellites, to study the highest-energy cosmic rays as well as their potential astrophysical sources.

All usually admitted principles of physics and properties of standard particles should thus be tested as far as possible: energy and momentum conservation, quantum mechanics, vacuum properties and stability, particle propagation in vacuum, quark confinement... Cosmology should consider these results. UHECR data and statistics are not yet enough for such purposes, but they already provide important information. 


\section{Further phenomenological considerations}

Assuming the VRF to be close to that naturally suggested by cosmic microwave background radiation $(\mathrm{CMB})$, the rest frames used in UHECR and other experiments will move at nonrelativistic speed with respect to the VRF. Then, an equation like (1) remains stable under standard Lorentz transformations between such frames except for corrections much smaller than the deformation term. Within the same approximation, these transformations naturally preserve the additivity and conservation of energy and momentum allowing for a simple phenomenological handling.

As pointed out in $[7,8]$ and further stressed in $[9,10]$, patterns with a VRF allow for new phenomena that would be forbidden in the presence of an exact space-time symmetry. This is the case for energy-dependent spontaneous decays. In the presence of exact Lorentz symmetry or of some deformed version of it without a privileged rest frame, stable (unstable) particles remain stable (unstable) at all energies. But in the presence of a VRF and of a negative deformation term like the $\Delta E$ from (1), the situation can be different as kinematical balances become energy-dependent.

In particular, it costs more and more energy, as $E$ increases, to split the deformation term given by (2). One has, for instance:

$$
\Delta E(p)=8 \Delta E(p / 2)
$$

so that, using always (1), the decay of a particle with momentum $p$ into two particles with momentum $p / 2$ with the same kinematics will cost an amount $3 \Delta E / 4$ in deformation energy. At high $E$, this cost in energy will preclude the decay if for instance: i) the particle and its decay products have the same value of $\alpha$; ii) the mass terms (that decrease with energy) cannot compensate the balance. If the values of $\alpha$ are not the same for all particles, those with lower $\alpha$ will decay into those with larger $\alpha$.

Thus, particles that are stable at low energy can become unstable at higher energy, and conversely. Similarly, if the energy and opposite momentum of a CMB photon are not enough to generate a suitable splitting of the $\Delta E$ of the incoming UHECR, the GZK mechanism becomes impossible as first pointed out in $[9,10]$. $E_{\text {trans }}$ is expected to be higher for nuclei as compared to the proton, but the deformation of the effective nuclear binding energy can be important and play a nontrivial role if new nuclear physics triggered by Lorentz contraction manifests itself below $E_{\text {trans }}$.

However, as discussed in Section 2, the deformed kinematics of the proton can have a value of $\alpha$ smaller than those of quarks and gluons, similar to nuclei as compared to the proton $[12,18]$. The values of $E_{\text {trans }}$ for the proton and nuclei would then be higher than expected from (1) with $\alpha=1$, and the existence of the GZK cutoff would still be compatible with a significant LSV at the Planck scale.

Furthermore, if the UHE proton has a smaller value of $\alpha$ than the photon, it can spontaneously decay by emitting a photon if the photon value of $\Delta E$ becomes larger than the proton mass term. If the photon has a lower $\alpha$ than the electron, it can in turn decay into an $\mathrm{e}^{+} \mathrm{e}^{-}$pair. Thus, even if the standard GZK cutoff is inhibited for protons by new physics, an alternative cutoff generated by LSV may appear in the same energy region [16, 18]. In this case, if the deformation of the photon or electron energy is to be compared with the mass term of the emitting proton or nucleus for such spontaneous decays, the observed mass composition of the UHE AUGER spectrum can naturally emerge, as heavier nuclei will start decaying at higher energies. 


\section{EPJ Web of Conferences}

\subsection{Other possibilities}

As stressed in Section 3, LSV is not the only violation of standard principles of physics that can potentially generate an alternative explanation to the observed fall of the UHECR spectrum in the presence of LSV and of a VRF.

The above described deformation of quantum mechanics can fake the GZK cutoff when the energy uncertainty $\Delta_{q} E \gtrsim\left[3 \Phi\left(p^{2}\right)\right]^{1 / 2} c$ becomes of the same order as the mass term $m^{2} c^{3}(2 p)^{-1}$ or the LSV deformation $\Delta E$. A very small violation of energy and momentum conservation can produce the same kind of effect. Unconventional interactions of UHECR with vacuum fluctuations at very short distance scales can be play a similar role if the internal structures of the UHE particle and of the (superbradyonic ?) vacuum are influenced by new physics generated at the Planck scale and beyond it, including important deviations from standard SQFT [22, 23].

Fluctuations of the vacuum structure at cosmic scale can also produce a UHECR flux suppression, especially if the conventional boson fields are not permanently condensed in vacuum as predicted by SQFT $[16,20]$ and if the vacuum is actually made of new constituents of matter (superbradyons) or similar nonstandard objects [9, 23].

Another possibility is that quarks and gluons cease to be confined at UHE due to the change in hadron internal structure. If this is the case, and if they cannot exist as free particles below some critical energy threshold in the VRF, quarks and gluons close to this critical energy can be part of the dark matter. Then, UHE hadron decays into such quarks and gluons can also fake the GZK cutoff.

These are just a few examples of mechanisms potentially able to produce effects faking the GZK cutoff in the presence of LSV with a VRF, and to fit the observed data. Obviously, further experimental and theoretical work is required.

It must also be kept in mind that, if the UHECR internal structure is modified by new physics, so are expected to be their interaction properties with matter at targets and detectors. Then, the measured UHECR spectrum and composition can be significantly distorted as compared to expectations from conventional signatures.

\section{LSV and neutrino physics at accelerators}

In September 2011, the OPERA collaboration (CERN Gran Sasso) reported [24] that muon neutrinos between a few $\mathrm{GeV}$ and more than $100 \mathrm{GeV}$ appeared to travel at a speed $c(1+\delta)$ with $\delta=(2.48 \pm 0.28) \times 10^{-5}$. Thus, a superluminal neutrino propagation seemed to have been observed.

For obvious reasons, the coherence of the approaches considered here concerning UHECR and deformations of standard particle properties also requires paying attention to much lower energies. The energy dependence of the possible mechanisms produced by new physics is a crucial issue for phenomenology. In particular, the OPERA result did not seem to fit with the patterns just described, as a strong LSV effect was manifesting itself at an energy scale too far below the UHE scales.

Now the claim of a possible superluminal neutrino has been withdrawn [43] after having been disavowed by a more recent experiment [44].

The consistency problems of the superluminal neutrino initially claimed by OPERA had been pointed out a few days after the OPERA announcement in our paper [25] and further developed the day after in $[26,46]$ : 
i) spontaneous neutrino decays, the superluminal neutrino being in particular able to emit electron-positron pairs [25, 46];

ii) to be able to decay by emitting this superluminal neutrino, the charged pion should present a similar critical speed anomaly. The anomaly would then propagate to hadrons and cosmic rays, in contradiction with experimental data $[25,26]$.

Taking the pion critical speed in vacuum to be equal to $c$, a trivial bound can be immediately obtained [26] before considering the precise values of lepton and neutrino masses:

$$
p_{\nu}^{2} \leq m_{\pi}^{2} c^{3}\left[\begin{array}{lll}
2 & \delta & c
\end{array}\right]^{-1}
$$

( $p_{\nu}=$ neutrino momentum, $m_{\pi}=$ pion mass $)$.

With $\delta=(2.48 \pm 0.28) \times 10^{-5}$, this result leads to $p_{\nu} \leq 20 \mathrm{GeV} / \mathrm{c}$, in clear contradiction with the OPERA data. $\delta=10^{-6}$ would yield $p_{\nu} \leq 100 \mathrm{GeV}$. Such bounds can be made stronger taking into account the muon mass [26].

Even if it may be argued that these estimates tacitly use a preferred reference frame, this does not seem to influence the above figures. The basic result on the consistency problems met by the OPERA superluminal neutrino does not depend on this issue, as the possible relevant corrections would in any case be smaller.

Consistency with SN1987A data was also a problem for the OPERA result [47], even if the energy scales involved were not the same.

Tests of LSV at accelerators were already considered in [48]. An updated approach should also pay attention to deviations from SQFT [22, 23]. Possible tests of other patterns at energies lower than UHE have been discussed in [49].

\section{Conclusion}

Observed cosmic rays have been seen to reach momenta corresponding to wavelengths as small as $\sim 10^{-25} \mathrm{~cm}$. This distance scale lies seven orders of magnitude beyond the present reach of accelerators and appears remarkably close to the Planck length.

Therefore, as already emphasized in [9, 37] and further discussed here, UHECR experiments provide a powerful and unique microscope directly focused on the Planck scale and beyond. No equivalent particle physics facility can be designed for this crucial exploration of the (possibly) ultimate laws of matter and space-time.

To further study the properties of the highest energy cosmic rays, long-term experimental programs including permanent Earth observatories like AUGER and satellite missions like JEM-EUSO [50] are required, allowing for much better statistics and precision combined with more detailed analyses and further theoretical developments.

Thus, UHECR experiments appear as the natural complement to cosmological CMB explorations [30, 31]. Cosmology being thus combined with the study of physics generated at the Plank scale and possibly beyond this scale.

All basic principles of standard particle physics should be tested as far as possible through UHECR experiments in an open-minded way. The present paper is just a preliminary strategic overview of the potentialities of such a research line.

\section{References}

[1] K.H. Kampert for the Pierre Auger Collaboration, Proceedings of ICRC 2011, Beijing August 2011, available at arXiv.org, arXiv:1207.4823 
[2] The Pierre Auger Collaboration, JCAP 04 (2012), 040, available at arXiv.org, arXiv: 1210.3602

[3] The Pierre Auger Collaboration, arXiv.org, arXiv:1210.3602

[4] P. Sokolsky, for the HiRes collaboration, ISVHECRI 2010, Batavia June-July 2010, available at arXiv.org, arXiv:1010.2690

[5] K. Greisen, Phys.Rev.Lett. 16 (1966), 748.

[6] G.T. Zatsepin and V.A. Kuz'min, JETP Letters 4, 78

[7] L. Gonzalez-Mestres, arXiv.org, arXiv:astro-ph/9606054

[8] L. Gonzalez-Mestres, contribution to the 28th International Conference on High Energy Physics, Warsaw 1966, available at arXiv.org, arXiv:hep-ph/9610474, and references therein.

[9] L. Gonzalez-Mestres, arXiv.org, astro-ph/9704017

[10] L. Gonzalez-Mestres, Proceedings of the 25th International Cosmic Ray Conference, Potchefstroomse Universiteit 1997, Vol. 6, p. 113. Available at arXiv.org, arXiv:physics/9705031

[11] L. Gonzalez-Mestres, Proc. Heidelberg 2000 Int. Symp. HE $\gamma$-Ray Astr., AIP Conf.Proc. 558 (2001), 874, available at arXiv.org, astro-ph/0011182.

[12] L. Gonzalez-Mestres, CRIS 2010 Proceedings, Nucl. Phys. B, Proc. Suppl. 212-213 (2011), 26, and references therein. The arXiv.org version arXiv:1011.4889 includes a relevant Post Scriptum.

[13] L. Gonzalez-Mestres, Proceedings of the January 1995 Moriond Workshop, Ed. Frontières, available at arXiv.org, arXiv:astro-ph/9505117

[14] L. Gonzalez-Mestres, Proceedings of the TAUP 1995 Conference, Nucl. Phys. Proc. Suppl. 48 (1996), 131, available at arXiv.org, arXiv:astro-ph/9601090

[15] See, for instance, S.K. Lamoreaux, Nature 416 (2002), 803, and references therein.

[16] L. Gonzalez-Mestres, 2011 Europhysics Conference on High Energy Physics, PoS EPSHEP2011 (2011) 390, and references therein.

[17] L. Gonzalez-Mestres, arXiv.org, arXiv:0802.2536

[18] L. Gonzalez-Mestres, CRIS 2008 Proceedings, Nucl. Phys. Proc. Suppl. 190 (2009), 191, available at arXiv.org, arXiv:0902.0994

[19] L. Gonzalez-Mestres, contribution to the Planck 2011 Conference, arXiv.org, arXiv:1110.6171

[20] L. Gonzalez-Mestres, Inv. Univ. Int. Conf., Paris 2009, AIP Conf.Proc. 1241 (2010), 1207. The arXiv.org version arXiv:0912.0725 contains a relevant Post Scriptum.

[21] L. Gonzalez-Mestres, arXiv.org, arXiv:1009.1853

[22] L. Gonzalez-Mestres, 2011 Europhysics Conference on High Energy Physics, PoS EPSHEP2011 (2011) 479, and references therein.

[23] L. Gonzalez-Mestres, Pre-Big Bang, fundamental Physics and noncyclic cosmologies, these Proceedings.

[24] The OPERA collaboration, arXiv.org, arXiv:1109.4897v1

[25] L. Gonzalez-Mestres, arXiv.org, arXiv:1109.6308

[26] L. Gonzalez-Mestres, arXiv.org, arXiv:1109.6630

[27] H. Poincaré, L'état actuel et l'avenir de la physique mathématique, 1904 St. Louis Conference, Bulletin des sciences mathématiques http://gallica.bnf.fr/ark:/12148/bpt6k486258r/f476.image 28, 30, and previous pa- 
pers by the same author.

[28] A. Einstein, Geometry und erfahrung, Preus. Akad. der Wissench., Sitzungsberichte, part I, p. 123 (1921). Translation, Geometry and experience, in Sidelights on relativity, Methuen, London 1922.

[29] V. Berezinsky1, M. Kachelriess, and A.Vilenkin, Phys.Rev.Lett. 79 (1997), 4302. Available at arXiv.org, arXiv:astro-ph/9708217

[30] Wilkinson Microwave Anisotropy Probe, http://map.gsfc.nasa.gov/

[31] Planck mission (European State Agency), http://sci.esa.int/sciencee/www/area/index.cfm?fareaid $=17$

[32] L. Gonzalez-Mestres, Pre-Conference AUGER Workshop of the 25th International Cosmic Ray Conference, Durban South-Africa, July 1997, arXiv.org, arXiv:physics/9706032

[33] L. Gonzalez-Mestres, Workshop on "Observing Giant Cosmic Ray Air Showers for > 10E20 eV Particles from Space", Univ. of Maryland, November 1997, AIP Conf. Proc. 433, 148 (1998), available at arXiv.org, arXiv:physics/9712047

[34] L. Gonzalez-Mestres, International Conference on Relativistic Physics and some of its Applications, Athens, June 1997, arXiv.org, arXiv:physics/9706022

[35] M. Froissart, Phys. Rev. 123, 1053 (1961).

[36] L. Gonzalez-Mestres, International Conference on Relativistic Physics and some of its Applications, Athens, June 1997, in Open Questions in Relativistic Physics, Ed. F. Selleri, Apeiron 1998. Available at arXiv.org, arXiv:physics/9709006

[37] L. Gonzalez-Mestres, ICRC 1999 Evening Workshop Session talk, arXiv.org, arXiv:physics/0003080, and references therein.

[38] L. Gonzalez-Mestres, Contribution to the I Symposium on European Strategy for Particle Physics, Orsay (France), January 30 - February 1st, 2006, arXiv.org, arXiv:hep$\mathrm{ph} / 0601219$, and references therein.

[39] T. Jacobson, S. Liberati and D. Mattingly, Nature 424 (2003), 1019. Available at arXiv.org, arXiv:astro-ph/0212190

[40] J. Wess, Lectures given at the 38. Internationale Universitätswochen für Kern- und Teilchenphysik, Schladming (Austria), January 1999

[41] L. Gonzalez-Mestres, arXiv.org, arXiv:0908.4070, and references therein.

[42] N.E. Mavromatos and R.J. Szabo, arXiv.org, arXiv:hep-th/9811116

[43] N. Yu. Agafonova et al., arXiv.org, arXiv:arXiv:1206.2488

[44] M. Antonello et al. (ICARUS Collaboration), arXiv.org, arXiv:1203.3433. See also [45].

[45] M. Antonello et al. (ICARUS Collaboration), arXiv.org, arXiv:1208.2629

[46] A.G. Cohen and S. Glashow, Phys. Rev. Lett. 107 (2011), 181803, available at arXiv.org, arXiv:1109.6562

[47] See, for instance, D. Fargion and D. D'Armiento, J. Phys. G: Nucl. Part. Phys. 39 (2012), 085002. Available at arXiv.org, arXiv:1109.5368

[48] L. Gonzalez-Mestres, EPS-HEP97, Jerusalem, August 1997, arXiv.org, arXiv:physics/9708028, and references therein.

[49] J. Ellis and N.E. Mavromatos, arXiv.org, arXiv:1111.1178

[50] JEM-EUSO, http://jemeuso.riken.jp/ 